\title{
EMPLOYMENT OF SECOND ORDER RULED SURFACES IN DESIGN OF SHEET BEAM GUNS
}

\author{
A. Krasnykh, Stanford Linear Accelerator Center, Menlo Park, California 94025
}

\begin{abstract}
A novel 3D method of sheet beam gun design has recently been developed. Second order ruled surfaces (SORS) can be used to define the geometry of the gun electrodes. The gun design process is made simpler if SORS are derived from analytical formulas. A proposed method is discussed and illustrated.
\end{abstract}

\section{INTRODUCTION}

High power RF source development is a field of activity that plays a dominant role in the development of modern accelerator technology. A modern trend is the development of multi and sheet beam RF-sources. Design of the high power sheet beam klystrons for different applications is a major research topic for the Klystron and Microwave Department at SLAC. The sheet beam approach can achieve higher peak and average power compared with traditional devices. Electron beam formation and transport though a klystron requires very good gun optics design. A 3D code is required for proper design of such a gun. The procedure of cathode, focusing electrode, and anode design is complicated in the 3D case. There are several sophisticated simulation codes used in the design of a sheet beam-gun. A method that has been employed at SLAC, uses, in sequence, Solid Edge, Direct CAD Interface Module, ICEM CFD, and Voyager packages. Solid Edge (or Solid Work) is a 3D solid modeling program where the initial gun geometry is created. The electron gun model is translated into a finite element grid with ICEM CFD software, using the Direct CAD Interface Module. The gun designer has to organize and prepare a gun model from the ICEM CFD grid, i.e. to clean up unneeded information, to extract curves and points, and to build surfaces to represent outer boundaries and vacuum volume.

To complete the gun design with required output parameters, the designer needs to make many iterations: to change initial geometry, to translate into ICEM CFD, to re-mesh, and so on. If the reader take into account that the gun geometry is $3 \mathrm{D}$, the gun design can be very time consuming work, even if the parametric models are used. The motivation of the present article is to show a method that simplifies the sheet beam gun design.

\section{MAJOR SHEET BEAM GUN PARAMETERS AND SECOND ORDER RULED SURFACES}

We need to find the gun electrode shapes for required beam current, beam energy, beam cross section, and gun voltage hold-off. Pierce theory tell us that the major optic parameters are cathode half angle, cathode radius, cathode area, and the Pierce angle for the slope of the focus electrode. A solution can be easily found for the axially symmetric case, i.e. for $2 \mathrm{D}$ gun geometry. It is difficult to find for a 3D case. Our experience shows that the gun geometry design is a more time consuming process. A designer often runs into the well-known problems of "surfaces don't match", "holes in geometry", "overlaps in surfaces", and "missing surfaces". In order to simplify a 3D sheet beam gun design we are proposing to restrict the field of the geometry exploration. Firstly, we would like to use a round or an elliptical cathode with a cylindrical surface. Secondly, we would like to employ SORS to define the shape of the gun focus electrode. A ruled surface is a surface that can be swept out by moving a line in space. We would like to consider more practical case: the beam from cathode has to have a compression in one plane only. If the Cartesian coordinate system (where $\mathrm{z}$ axis is for a beam direction) is taken into account, then there are two focus electrode Pierce angles, for $\mathrm{y}$ (compression) and $\mathrm{x}$ (non compression) planes. They are a result of SORS representation. The view on the cathode and SORS surface from $\mathrm{z}$ axis will be smooth and there are no sharp corners or transitions. Finally, we are going to employ the simple mathematical functions that describe the sheet beam gun electrodes.

A diagram shown in Fig. 1 illustrates the proposed idea. A quarter-section of the cathode and an initial part of a focus electrode are shown with a beam. The cathode surface is an elliptical cylinder with semi axis oa and $\boldsymbol{o c}$. The oac emitter surface is created by the cylinder cutout with radius $\boldsymbol{R} \boldsymbol{c}$. If $\boldsymbol{o a}=\boldsymbol{o c}$ we obtain the round cathode with a cylinder cutout emitter. The abcd surface is a focus electrode surface. This SORS surface is formed by sweeping a straight $\boldsymbol{a} \boldsymbol{b}$-line along $\boldsymbol{a c}$-curve. Angles $\theta y$ and $\theta x$ are Pierce angles for compression and no compression planes. 


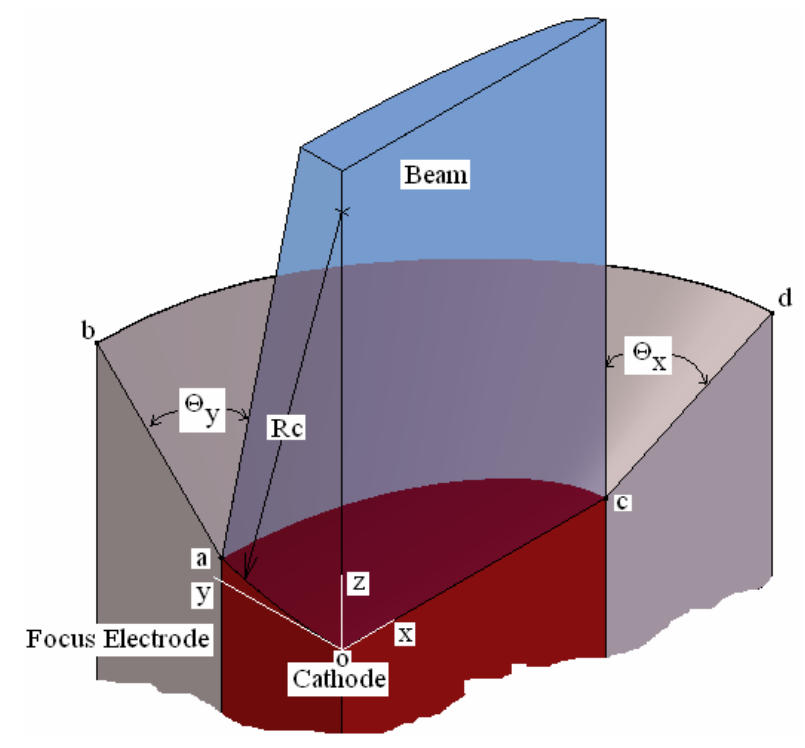

Fig. 1 Quarter-section of initial part of gun model

Mathematical formulas for the emitter surface representation in polar coordinate system are

$$
\begin{aligned}
& x(\varphi)=r(\varphi) \cos (\varphi) \\
& y(\varphi)=r(\varphi) \sin (\varphi) \\
& z(\varphi)=\frac{b}{\sin (\alpha)} \pm \sqrt{\left(\frac{b}{\sin (\alpha)}\right)^{2}-(r(\varphi) \sin (\varphi))^{2}} \\
& r(\varphi)=\sqrt{\frac{(a b)^{2}}{(b \cos (\varphi))^{2}+(a \sin (\varphi))^{2}}}
\end{aligned}
$$

where $\varphi$ is a polar angle, and $\alpha$ is a cathode half angle, $\boldsymbol{a}=\boldsymbol{o c}, \boldsymbol{b}=\boldsymbol{o a}, \boldsymbol{b}=\boldsymbol{R} \boldsymbol{c} * \sin (\alpha)$. Usually $\boldsymbol{a}, \boldsymbol{b}$, and $\boldsymbol{R} \boldsymbol{c}$ are the major sheet beam gun parameters. The $\boldsymbol{a c}$-curve may be the SORS guiding curve. The gap $\boldsymbol{\delta}$ between outer emitter and inner focus electrode curves may be easily taken into account if the sheet beam gun aberration is a subject for consideration.

Similar formulas may be employed for the outer guiding $\boldsymbol{b} \boldsymbol{d}$-curve except $\mathrm{z}(\varphi)$ function

$$
\begin{aligned}
& x(\varphi)=r(\varphi) \cdot \cos (\varphi) \\
& y(\varphi)=r(\varphi) \cdot \sin (\varphi) \\
& z(\varphi)=h_{1}+k_{1} \cdot(\sin (\varphi))^{2}+m_{1} \cdot(\sin (2 \varphi))^{2} \\
& r(\varphi)=\sqrt{\frac{\left(a_{1} \cdot b_{1}\right)^{2}}{\left(b_{1} \cdot \cos (\varphi)\right)^{2}+\left(a_{1} \cdot \sin (\varphi)\right)^{2}}}
\end{aligned}
$$

where a few parameters $(\boldsymbol{a} \mathbf{1}, \boldsymbol{b} \mathbf{1}, \boldsymbol{h} \mathbf{1}, \boldsymbol{k} \mathbf{1}$, and $\mathbf{m} \mathbf{1})$ are responsible for the gun Pierce angles $\theta y$ and $\theta x$.
The form of a $\mathbf{z}(\varphi)$ function (a function of axial modulation) will be discussed later after we complete a description of the second part of the focus electrode (see Fig. 2). The outer surface of the sheet beam gun focus electrode is a right cylinder with $\boldsymbol{R} \boldsymbol{f e}$ radius for most common practical cases. This radius will determine the voltage hold off relative to the anode housing.

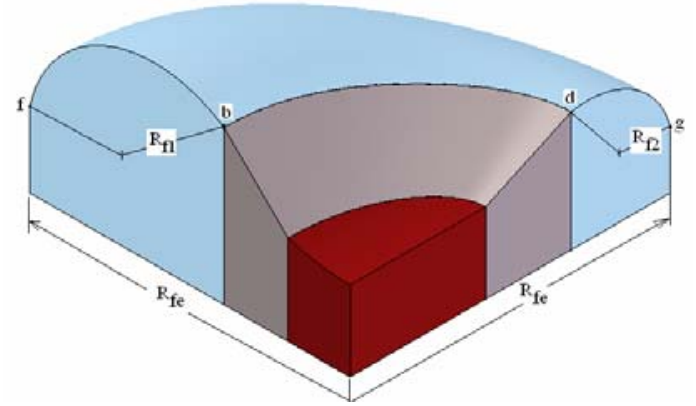

Fig. 2 Quarter model of cathode with first and second parts of focus electrode

Focus electrode radii $\boldsymbol{R} \boldsymbol{f} \mathbf{1}$ and $\boldsymbol{R} \boldsymbol{f} 2$ turn out to be not equal for the elliptical cathode. The outer surface of the focus electrode is a part of a toroidal surface. The electric field enhancement at the focus electrode surface for the compression and no compression planes is different. To minimize the electric gradient we will require that the derivative on the boundary between first and second part of the focus electrode surfaces is zero. The same condition we employ for the boundary where a toroidal surface mates with the outer cylinder surface. Third guiding curve is described in a similar manner but with the new coefficients: a2, $b 2, h 2, k 2$, and $m 2$-- where $a \mathbf{2}=b 2=R f e$, and $h 2$, $\boldsymbol{k} \mathbf{2}$, and $\boldsymbol{m} \mathbf{2}$ are parameters for the second part of the focus electrode. The required surfaces may be easily created in Solid Edge where the table curve representation is available (the same feature is available in Solid Works too). We shall emphasize that the designer works (varies) practically with a limited number of the geometry parameters. The CAD program automatically calculates the rest of the parameters to create a model according to the above restrictions i.e. the changing of one gun parameter requires a new set of the sheet beam gun parameters automatically. One gun model may be easily transformed to a similar one by a modification of a few gun parameters. The difficulty with "surfaces don't match", "holes in geometry", "overlaps in surfaces" etc. is eliminated.

So, the proposed method for sheet beam gun design defines the main gun surfaces. The focus electrode surfaces are simple, smooth, and have no sharp transitions. Because simplest mathematical 
formulas are employed to describe the electrode surfaces, they are easy to manufacture.

\section{EXAMPLE: RESULT OF SHEET BEAM GUN DESIGN}

Let us consider how this method works for the design of a sheet beam gun with the following specification: beam voltage is $250 \mathrm{kV}$, beam current is $94 \mathrm{~A}$, cathode is elliptical, and cathode width and height are $2 \mathrm{a}=50.8 \mathrm{~mm}$ and $2 \mathrm{~b}=23.2 \mathrm{~mm}$ accordingly. The outer beam cross section at crossover should be no more than 2.4 x $50 \mathrm{sq} \mathrm{mm}$. The cathode and focus electrode should fit in the 5045 SLAC klystron anode housing. The present article will not cover a design of the beam anode entrance and it is assumed to be given.

After several cycles it was found that $\alpha=18^{\circ}$ $(\mathrm{Rc}=37.54 \mathrm{~mm}), 2 \mathrm{a} 1=82.2 \mathrm{~mm} 2 \mathrm{~b} 1=47.2 \mathrm{~mm}$, $\mathrm{h} 1=7.46 \mathrm{~mm}, \mathrm{k} 1=6.39 \mathrm{~mm}, \mathrm{~m} 1=0.96 \mathrm{~mm}$, 2a2 $=2 \mathrm{~b} 2=120 \mathrm{~mm}, \mathrm{~h} 2=-4.39 \mathrm{~mm}, \mathrm{k} 2=3.15 \mathrm{~mm}$ and $\mathrm{m} 2=0.47 \mathrm{~mm}$ will meet the sheet beam gun spec. Results of the sheet beam gun design are shown in Fig. 3.

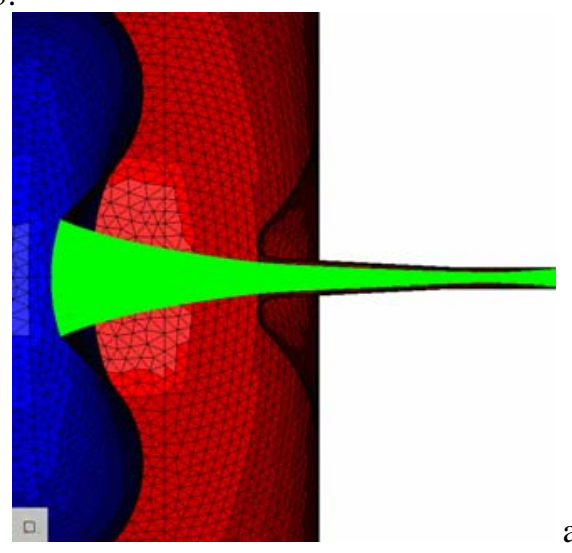

a)

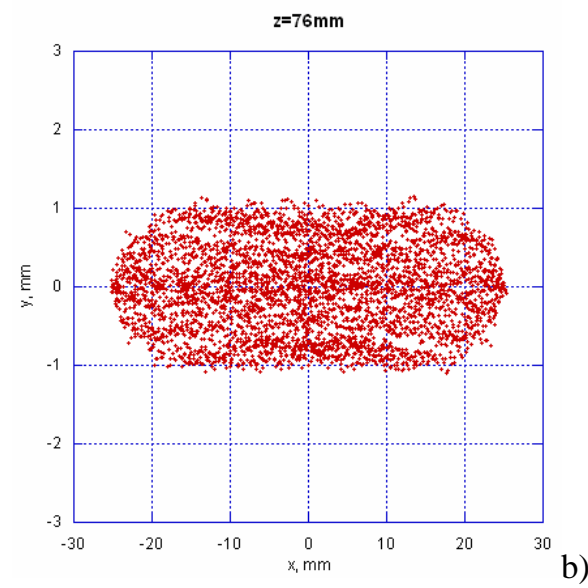

Fig. 3 Result of $250 \mathrm{kV}, 94 \mathrm{~A}$ sheet beam gun design: a) side view, b) beam cross section near crossover
Our experience shows that $\mathbf{z}(\varphi)$ function (a function of axial modulation) for a focus electrode may be chosen in more common case: $z(\varphi)=h_{i}+k_{i} \cdot(\sin (\varphi))^{n}+m_{i} \cdot(\sin (2 \varphi))^{m}$ where $\mathbf{n}$ and $\mathbf{m}$ are the gun parameters too. For high perveance guns those parameters were used to reduce the gun aberration or so called "butterfly effect” that is shown in Fig. 4.

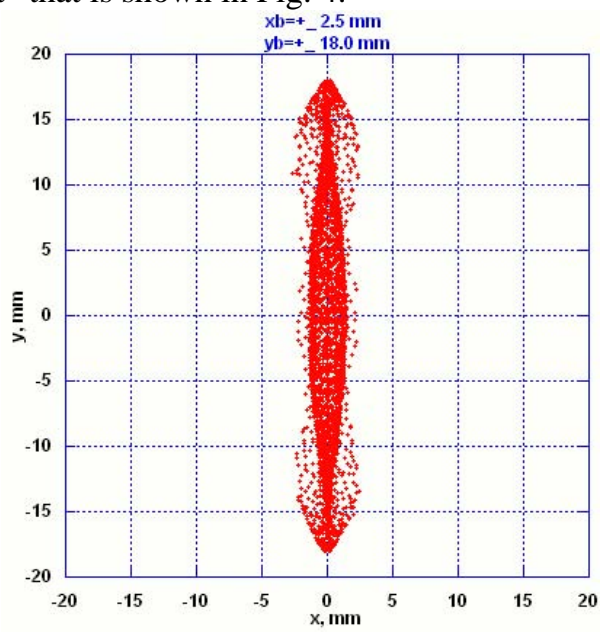

Fig. 4 Example for another gun design: a beam cross section in the middle of tuning process is shown. Here the different electrons undergo different focusing and there is a strong aberration effect

The effect may be sufficiently suppressed if the sheet beam gun optics is tuned by the above described method and upgraded axial modulation function is employed.

\section{CONCLUSION}

A sheet beam gun design method is presented. The focus electrode contains the second order ruled surface as a main part of the gun optics. The cathode and focus electrode faces are simply mathematically expressed. The gun model is parametrically described by an array of gun coefficients and may be easily tuned for the required specification. Another benefit of the proposed method is simplicity of the gun electrode manufacture.

\section{ACKNOWLEDGEMENTS}

This work was stimulated in the SLAC Klystron and Microwave Dept and discussed with my coworkers many times. Dr. G. Caryotakis, R. Phillips and G. Scheitrum were primary end users of results.

Work supported by the U.S. Department of Energy under contract number DE-AC0376SF00515 